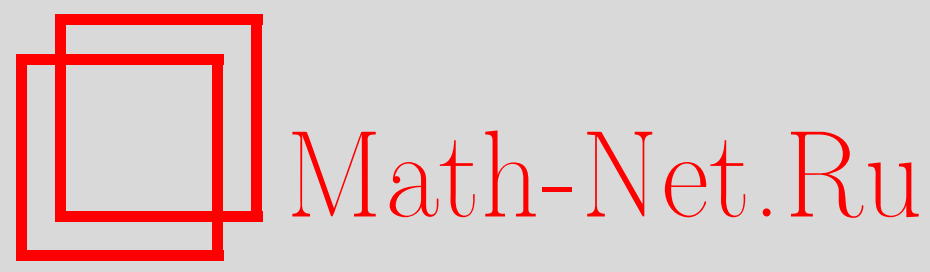

А. Е. Микушева, О сходимости вполне сумм отрицательно ассоциированных случайных величин, Матем. заметкu, 2000, том 68, выпуск 3, 411-420

DOI: https://doi.org/10.4213/mzm958

Использование Общероссийского математического портала Math-Net.Ru подразумевает, что вы прочитали и согласны с пользовательским соглашением http://www . mathnet.ru/rus/agreement

Параметры загрузки:

IP : 52.87 .193 .239

26 апреля 2023 г., 12:15:22

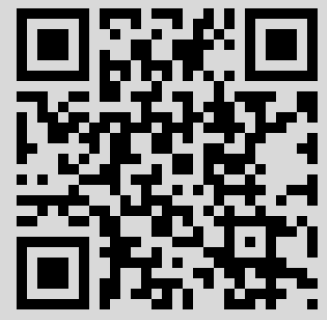




\title{
О СХОДИМОСТИ ВПОЛНЕ СУММ ОТРИЦАТЕЛЬНО АССОЦИИРОВАННЫХ СЛУЧАЙНЫХ ВЕЛИЧИН
}

\section{А. Е. Микушева}

\begin{abstract}
Эта работа обобщает результаты о сходимости вполне в законе больших чисел для подпоследовательностей на случай отрицательно ассоциированных неодинаково распределенных случайных величин.
\end{abstract}

Библиографоия: 13 названий.

1. Введение. Предельному поведению сумм независимых и слабо зависимых случайных величин посвящена обширная литература. В данной работе исследуется асимптотическое поведение сумм случайных слагаемьх в схеме серий. Точнее говоря, нас будет интересовать сходимость вполне (complete convergence), введенная в работах П. Л. Хсу, Г. Роббинса [1] и П. Эрдоша [2].

ОПРЕДЕЛЕНИЕ. Последовательность действительньх случайных величин $\left\{Y_{j}\right.$, $j \geqslant 1\}$ называется сходящейся вполне $\kappa 0$, если $\sum_{j=1}^{\infty} \mathrm{P}\left\{\left|Y_{j}\right|>\varepsilon\right\}<\infty$ для любого $\varepsilon>0$.

Эрдош [2] доказал, что если $\left\{X_{j}, j \geqslant 1\right\}$ - последовательность независимых одинаково распределенных центрированных случайных величин и $S_{n}=\sum_{j=1}^{n} X_{j}$, то сходимость вполне к 0 последовательности $\left\{S_{n} / n, n \geqslant 1\right\}$ равносильна конечности второго момента величины $X_{j}$.

Этот результат можно рассматривать с нескольких точек зрения: во-первых, как результат о новом виде сходимости, которая (по лемме Бореля-Кантелли) влечет за собой сходимость почти наверное, во-вторых, как оценку скорости сходимости в усиленном законе больших чисел, и наконец, как усиленньй закон больших чисел в схеме серий. А именно, рассматривается набор независимых одинаково распределенных центрированных случайных величин $\left\{X_{k, l}, 1 \leqslant l \leqslant k, k \geqslant 1\right\}, S_{k}=\sum_{l=1}^{k} X_{k, l}$; тогда теорема Эрдоша [2] как раз устанавливает необходимое и достаточное условие для сходимости $S_{k}$ к 0 почти наверное.

В дальнейшем появился цельй ряд обобщений этого результата; упомянем лишь некоторые из них. Так Т. Ху, Ф. Мориц и Р. Л. Тэйлор [3] в 1992 году получили необходимые и достаточные условия для сходимости вполнепоследовательности $\left\{S_{n} / n^{p}, n \geqslant 1\right\}$, где $1 / 2<p \leqslant 1$.

Асмуссен и Куртц [4] рассмотрели вопрос о сходимости вполне к 0 последовательности $\left\{S_{N_{k}} / N_{k}, k \geqslant 1\right\}$, где $N_{k}-$ строго возрастающая последовательность натуральных 
чисел, удовлетворяющая некоторым техническим условиям (в частности, она должна расти не медленнее степенной, но не быстрее показательной последовательностей). Далее А. Гут [5], [6] дополнил результат Асмуссена и Куртца, рассмотрев иные классы последовательностей $N_{k}$, а также использовав более общую нормировку $\left\{S_{N_{k}} / N_{k}^{p}, k \geqslant 1\right\}$.

Тут следует сделать несколько замечаний. Во-первых, совокупность результатов Асмуссена, Куртца [4] и Гута [5], [6], вообще говоря, не покрывает все множество строго возрастающих последовательностей $N_{k}$. Во-вторых, в работах Гута [5], [6] рассматривается два класса последовательностей $N_{k}$, условно назовем их "быстро растущие" и "медленно растущие". Для "медленно растущих" последовательностей им получены условия, являющиеся необходимьми и достаточньми. В то же время, для “быстро растущих" последовательностей достаточные условия, предложенные упомянутым автором, вообще говоря, не являются необходимыми, как показали в своей работе А. Кусмазевска и Д. Шиналь [7].

Также обобщения теоремы Эрдоша рассматривались в работах [8]-[10].

Все перечисленные вьше результаты имеют дело с последовательностями независимых случайных величин, мы же обратимся к отрицательно ассоциированным. Понятие отрицательно ассоциированной последовательности случайных величин введено в 1983 году К. Жоаг-Дев и $\Phi$. Прошаном [11]. Напомним, что любое семейство независимых случайных величин автоматически будет отрицательно ассоциированным. Интересные примеры величин, обладающих отрищательной ассоциированной зависимостью, можно найти в [11].

Аналог теоремы Эрдоша для отрицательно ассоциированных случайных величин был получен в 1996 году Ч. Су [12].

Целью данной работы будет обобщение результата Ч. Су [12] и получение необходимых и достаточных условий для сходимости вполне к 0 нормированных сумм отрицательно ассоциированных случайных величин $\left\{S_{N_{k}} / N_{k}^{p}, k \geqslant 1\right\}, 1 / 2<p \leqslant 1$, для достаточно широкого класса последовательностей $N_{k}$.

Всюду далее мы рассматриваем действительные случайные величины, заданные на некотором вероятностном пространстве $(\Omega, F, \mathrm{P})$.

\section{2. Основные результаты.}

ОПРЕДЕЛЕНИЕ. Случайные величины $X_{1}, \ldots, X_{n},(n \geqslant 2)$ называются отрицательно ассоциированными, если для любых двух непустых непересекающихся множеств $A$ и $B \subset\{1, \ldots, n\}$ и пары покоординатно невозрастающих (неубьвающих) функций $f$ и $g$ имеет место неравенство

$$
\operatorname{cov}\left(f\left(X_{i}, i \in A\right), g\left(X_{j}, j \in B\right)\right) \leqslant 0,
$$

когда ковариация существует.

ОПРЕДЕЛЕниЕ. Массив случайных величин $\left\{X_{k, l}, 1 \leqslant l \leqslant N_{k}, k \geqslant 1\right\}$ называется слабо мажсорируемым случайной величиной $X$, если для некоторого $\beta>0$ и для всех $t>0$ и $k \geqslant 1$ выполняется условие

$$
\frac{1}{N_{k}} \sum_{l=1}^{N_{k}} \mathrm{P}\left\{\left|X_{k, l}\right|>t\right\} \leqslant \beta \mathrm{P}\{|X|>t\} .
$$


Это условие, предложенное Гутом [6], строго слабее условия стохастической мажорируемости, т.е. $\mathrm{P}\left\{\left|X_{k, l}\right|>t\right\} \leqslant \mathrm{P}\{|X|>t\}$ для всех $t$. Соответствующие примеры приведены в работе [6].

Введем в рассмотрение строго возрастающую непрерывную функцию $\psi:[0, \infty) \rightarrow$ $[0, \infty)$. Будем считать, что

$$
\psi(k)=N_{k} \in \mathbb{N} \quad \text { для } k \in \mathbb{N}
$$

и обратная к ней $\psi^{-1}(\cdot)$ является абсолютно непрерывной с производной $\gamma$.

ТЕОРемА 2.1. Пусть массив чентрированных случайных величин $\left\{X_{k, l}, 1 \leqslant\right.$ $\left.l \leqslant N_{k}, k \geqslant 1\right\}$ слабо мажсорируется случайной величиной $X$. Пусть к тому же, для любого фиксированного $k$ величины $\left\{X_{k, l}, 1 \leqslant l \leqslant N_{k}\right\}$ являются отричательно ассочиированными. Предположсм, что $N_{k}$ задается введенной выше функцией $\psi$ согласно (1).

Пусть также для заданного $p \in(1 / 2,1]$ выполняется один из двух наборов условий:

а) для некоторых $C, \theta>0$ и всех $y>1, x>0$

$$
\begin{aligned}
& \gamma(x y) \leqslant C y^{\theta} \gamma(x), \\
& \varliminf_{k \rightarrow \infty} \frac{\sum_{l=1}^{k} N_{l}}{N_{k+1} k^{\delta}}>0 \quad \text { при некотором } \delta>0 \text {, } \\
& \sum_{k=1}^{\infty} N_{k} \mathrm{P}\left\{|X| \geqslant \varepsilon N_{k}^{p}\right\}<\infty \quad \text { для всех } \varepsilon>0
\end{aligned}
$$

б) справедливо

$$
\begin{gathered}
\varlimsup_{k \rightarrow \infty} \frac{\sum_{l=1}^{k} N_{l}}{N_{k+1}}<\infty, \\
\mathrm{E}|X|^{1 / p}<\infty
\end{gathered}
$$

Тогда, полагая $S_{k, n}=\sum_{l=1}^{n} X_{k, l}, n=1, \ldots, N_{k}$, имеем

$$
\sum_{k=1}^{\infty} \mathrm{P}\left\{\max _{1 \leqslant n \leqslant N_{k}}\left|S_{k, n}\right| \geqslant \varepsilon N_{k}^{p}\right\}<\infty \quad \text { nри всех } \varepsilon>0 .
$$

ЗАмЕЧАнИЕ 1 . Если в теореме 2.1 положить $\psi(x)=x$, т. е. $N_{k}=k$, то, принимая во внимание, что условие $\sum_{k=1}^{\infty} k \mathrm{P}\left\{|X|>k^{p}\right\}<\infty$ равносильно условию $\mathrm{E}|X|^{2 / p}<\infty$, получаем в качестве следствия теорему, доказанную для независимых случайных величин Ху, Морицем и Тэйлором [3].

ЗАмечАниЕ 2 . Теорема 2.1 в условиях пункта б) обобщает теорему Гута [6, теорема 4.2] на случай отрицательно ассоциированных случайных величин. 
ЗАмЕчАнИЕ 3. Примером последовательности, удовлетворяющей условиям (2) и (3), может служить $\psi(x)=x^{\alpha}$, т.е. $N_{k}=\left[k^{\alpha}\right], \alpha \geqslant 1$, а последовательности, удовлетворяющей $(5), \psi(x)=2^{x}, N_{k}=2^{k}$ и $\psi(x)=2^{2^{x}}, N_{k}=2^{2^{k}}$. Теорема 2.1 не охватьвает все множество строго возрастающих последовательностей $N_{k}$, впрочем, не существует до сих пор подобной теоремы и для независимых слагаемых.

Следующая теорема дает необходимое условие для вьполнения (7).

Теорема 2.2. Пусть $\left\{X_{k, l}, 1 \leqslant l \leqslant N_{k}\right\}$ - отрицательно ассоичированнье случайные величины при любом $k \geqslant 1$. Тогда из (7) следует

$$
\sum_{k=1}^{\infty} \sum_{l=1}^{N_{k}} \mathrm{P}\left\{\left|X_{k, l}\right| \geqslant \varepsilon N_{k}^{p}\right\}<\infty \quad \text { для любого } \varepsilon>0 .
$$

В частности, если $\left\{X, X_{k, l}, 1 \leqslant l \leqslant N_{k}, k \geqslant 1\right\}$ - одинаково распределенные случайные величины, то из (7) следует (4).

ЗАмЕчАНИЕ 4. Назовем последовательность $N_{k}$, удовлетворяющую условиям (1), (2) и (3), "медленно растущей", а удовлетворяющую (5), “быстро растущей". Видим, что для "медленно растущих" последовательностей случайных величин условие (4) является необходимым и достаточным для выполнения (7), в то время как для “быстро растущих" необходимое и достаточное условия не совпадают.

ЗАмЕчАниЕ 5. В литературе часто используется другое определение “быстро растуших" последовательностей:

$$
\varliminf_{k \rightarrow \infty} \frac{N_{k+1}}{N_{k}}>1
$$

которое является более ограничительным, чем (5). Действительно, оно означает, что существует $C>1$ такое, что $N_{k+1}>C N_{k}$ для любого $k$. В частности, $N_{l}<C^{-(k-l)} N_{k}$ при $l<k$ или $\sum_{l \leqslant k} N_{l}<N_{k} \sum_{l \leqslant k} C^{-(k-l)} \leqslant C N_{k+1} /(C-1)$, откуда следует (5).

Рассмотрим величины

$$
S_{n}^{(k)}=S_{k, N_{k}}-X_{k n}=\sum_{\substack{l=1 \\ l \neq n}}^{N_{k}} X_{k l}
$$

Сформулируем теорему, являющуюся обобщением результата Су [12] на достаточно широкий класс последовательностей $N_{k}$.

Теорема 2.3. Пусть задан массив $\left\{X, X_{k, l}, 1 \leqslant l \leqslant N_{k}, k \geqslant 1\right\}$ одинаково распределенных, центрированных, для любого фиксированного $k$ отрицательно ассоииированных случайных величин, $1 / 2<p \leqslant 1$. Пусть $\left\{N_{k}\right\}-$ строго возрастающая последовательность натуральных чисел, удовлетворяющая условиям (1), (2) и (3). Тогда условие (4) является необходимым и достаточным для выполнения

$$
\sum_{k=1}^{\infty} \mathrm{P}\left\{\max _{1 \leqslant n \leqslant N_{k}}\left|S_{n}^{(k)}\right| \geqslant \varepsilon N_{k}^{p}\right\}<\infty \quad \text { для всех } \varepsilon>0 .
$$


3. Доказательства. Буквой $C$ мы будем обозначать далее константы, вообще говоря, различные для разных формул.

Нам потребуются следующие утверждения.

Лемма 3.1 [6]. Пусть массив случайных величин $\left\{X_{k, l}, 1 \leqslant l \leqslant N_{k}, k \geqslant 1\right\}$ слабо мажсорируется величиной $X$. Пусть $r>0, A>0$,

$$
\begin{gathered}
X_{k, l}(A)=\left|X_{k, l}\right| \cdot I_{\left\{\left|X_{k, l}\right|<A\right\}}+A I_{\left\{\left|X_{k, l}\right| \geqslant A\right\}}, \quad X_{k, l}^{*}(A)=X_{k, l} \cdot I_{\left\{\left|X_{k, l}\right| \geqslant A\right\}}, \\
X(A)=|X| \cdot I_{\{|X|<A\}}+A I_{\{|X| \geqslant A\}}, \quad X^{*}(A)=X \cdot I_{\{|X| \geqslant A\}} .
\end{gathered}
$$

Tогда

$$
\frac{1}{N_{k}} \sum_{l=1}^{N_{k}} \mathrm{E}\left(X_{k l}(A)\right)^{r} \leqslant \beta \mathrm{E}(X(A))^{r}, \quad \frac{1}{N_{k}} \sum_{l=1}^{N_{k}} \mathrm{E}\left|X_{k l}^{*}(A)\right|^{r} \leqslant \beta \mathrm{E}\left|X^{*}(A)\right|^{r} .
$$

Будем использовать также максимальное неравенство, полученное Су, Зао и Ванг.

ЛЕмма 3.2 [13]. Пусть $m \geqslant 2 u\left\{Z_{i}, i \geqslant 1\right\}$ - последовательность отрицательно ассочиированных случайных величин $c \mathrm{E} Z_{i}=0 u \mathrm{E}\left|Z_{i}\right|^{m}<\infty$. Тогда существует константа $A_{m}>0$ такая, что

$$
\mathrm{E} \max _{1 \leqslant k \leqslant n}\left|\sum_{l=1}^{k} Z_{l}\right|^{m} \leqslant A_{m}\left(\left(\sum_{l=1}^{k} \mathrm{E} Z_{l}^{2}\right)^{m / 2}+\sum_{l=1}^{k} \mathrm{E}\left|Z_{l}\right|^{m}\right) .
$$

ЛЕмма 3.3. Условие (4) әквивалентно следующему:

$$
\mathrm{E} g\left(|X / \varepsilon|^{1 / p}\right)<\infty \quad \text { для любого } \varepsilon>0,
$$

где $g(x)=\sum_{N_{k} \leqslant x} N_{k}$. Из (3) и (4) следует (6), а (5) и (6) влекут (4). В частности, из условий теоремы 2.1 в обоих пунктах выполняется $u$ (4), $u$ (6).

ДокАЗАТЕЛЬСТвО. Нетрудно видеть, что

$$
\sum_{k=1}^{\infty} N_{k} \mathrm{P}\left\{|X| \geqslant \varepsilon N_{k}^{p}\right\}=\sum_{j=1}^{\infty} \mathrm{P}\left\{j \leqslant\left(\frac{|X|}{\varepsilon}\right)^{1 / p}<j+1\right\} \sum_{N_{k} \leqslant j} N_{k}=\mathrm{E} g\left(|X / \varepsilon|^{1 / p}\right),
$$

откуда следует эквивалентность (4) и (9). С учетом соотношения $g\left(N_{k}\right)=\sum_{l=1}^{k} N_{l}$ получаем второе утверждение леммы.

ЛЕмма 3.4. Пусть функиия

$$
G(x)=\int_{0}^{\psi^{-1}(x)} \psi(t) d t=\int_{0}^{x} t \gamma(t) d t .
$$

Тогда $G(x)-x \leqslant g(x) \leqslant G(x)+x$.

Таким образом, из (4) и (6) вытекает, что $\mathrm{E} G\left(|X|^{1 / p} \lambda\right)<\infty$ для любого $\lambda>0$, причем

$$
\mathrm{E} G\left(|X|^{1 / p} \lambda\right)=\frac{\lambda^{2}}{p} \int_{0}^{\infty} F(x) \gamma\left(\lambda x^{1 / p}\right) x^{2 / p-1} d x<\infty
$$

əде $F(t)=\mathrm{P}\{|X|>t\}$. 
ДоКАЗАТЕЛЬСТвО. Имеем

$$
g(x) \leqslant \sum_{k: k+1 \leqslant \psi^{-1}(x)} \psi(k)+N_{k^{*}} \leqslant \sum_{k: k+1 \leqslant \psi^{-1}(x)} \int_{k}^{k+1} \psi(t) d t+x \leqslant G(x)+x ;
$$

здесь $k^{*}=\max \left\{k: N_{k} \leqslant x\right\}$. Аналогичными выкладками приходим ко второму неравенству. Выражение (10) получается интегрированием по частям.

ДОКАЗАТЕЛЬСТВО ТЕОРЕМЫ 2.1. Произведем урезание случайных величин:

$$
\begin{gathered}
Y_{k, l}=X_{k, l} \cdot I_{\left\{\left|X_{k, l}\right| \leqslant N_{k}^{p}\right\}}+N_{k}^{p} \cdot I_{\left\{X_{k, l} \geqslant N_{k}^{p}\right\}}-N_{k}^{p} \cdot I_{\left\{X_{k, l} \leqslant-N_{k}^{p}\right\}}, \quad S_{k, n}^{*}=\sum_{l=1}^{n} Y_{k, l}, \\
Y_{k}=X \cdot I_{\left\{|X| \leqslant N_{k}^{p}\right\}}+N_{k}^{p} \cdot I_{\left\{X \geqslant N_{k}^{p}\right\}}-N_{k}^{p} \cdot I_{\left\{X \leqslant-N_{k}^{p}\right\}}, \quad X_{k}=|X| \cdot I_{\left\{|X| \leqslant N_{k}^{p}\right\}} .
\end{gathered}
$$

Поскольку $Y_{k, l}$ являются неубывающими функциями от $X_{k, l}$, то величины $\left\{Y_{k, l}, 1 \leqslant\right.$ $\left.l \leqslant N_{k}\right\}$ для любого $k$ обладают свойством отрицательной ассоциированности.

Заметим, что

$$
\mathrm{P}\left\{\exists n: S_{k, n}^{*} \neq S_{k, n}\right\} \leqslant \beta N_{k} \mathrm{P}\left\{|X| \geqslant N_{k}^{p}\right\} .
$$

Из леммы 3.3 следует вьполнение условия (4), поэтому достаточно доказать

$$
\sum_{k=1}^{\infty} \mathrm{P}\left\{\max _{1 \leqslant n \leqslant N_{k}}\left|S_{k, n}^{*}\right| \geqslant \varepsilon N_{k}^{p}\right\}<\infty
$$

Принимая во внимание центрированность и слабую можорируемость случайных величин, легко видеть, что

$$
\begin{aligned}
& \max _{1 \leqslant n \leqslant N_{k}} \frac{\left|\mathrm{E} S_{k, n}^{*}\right|}{N_{k}^{p}} \leqslant \sum_{l=1}^{N_{k}} \frac{\left|E Y_{k, l}\right|}{N_{k}^{p}} \leqslant \sum_{l=1}^{N_{k}} \mathrm{P}\left\{\left|X_{k, l}\right|>N_{k}^{p}\right\}+\sum_{l=1}^{N_{k}} \frac{\left|E X_{k, l} \cdot I_{\left\{\left|X_{k, l}\right|>N_{k}^{p}\right\}}\right|}{N_{k}^{p}} \\
& \leqslant \beta N_{k} \mathrm{P}\left\{|X|>N_{k}^{p}\right\}+\beta \frac{\mathrm{E}|X| \cdot I_{\left\{|X|>N_{k}^{p}\right\}}}{N_{k}^{p-1}} \leqslant \beta N_{k} \mathrm{P}\left\{|X|>N_{k}^{p}\right\} \\
& \quad+\beta \frac{N_{k}^{p(1-1 / p)} \mathrm{E}|X|^{1 / p} I_{\left\{|X|>N_{k}^{p}\right\}}}{N_{k}^{p-1}}=\beta N_{k} \mathrm{P}\left\{|X|>N_{k}^{p}\right\}+\beta \mathrm{E}|X|^{1 / p} I_{\left\{|X|>N_{k}^{p}\right\}} .
\end{aligned}
$$

По лемме 3.3 вьполняются условия (6) и (4); первое из них дает $\mathrm{E}|X|^{1 / p} I_{\left\{|X|>N_{k}^{p}\right\}} \rightarrow 0$ при $k \rightarrow \infty$, а второе влечет соотношение $N_{k} \mathrm{P}\left\{|X|>N_{k}^{p}\right\} \rightarrow 0$ при $k \rightarrow \infty$. Следовательно,

$$
\max _{1 \leqslant n \leqslant N_{k}} \frac{\left|\mathrm{E} S_{k, n}^{*}\right|}{N_{k}^{p}} \rightarrow 0 \quad(k \rightarrow \infty) .
$$

Таким образом, для доказательства теоремы достаточно показать сходимость ряда

$$
\sum_{k=1}^{\infty} \mathrm{P}\left\{\max _{1 \leqslant n \leqslant N_{k}}\left|S_{k, n}^{*}-\mathrm{E} S_{k, n}^{*}\right| \geqslant \frac{\varepsilon}{2} N_{k}^{p}\right\}
$$


Применив лемму 3.2 и неравенство Чебьшева, имеем

$$
\mathrm{P}\left\{\max _{1 \leqslant n \leqslant N_{k}}\left|S_{k, n}^{*}-\mathrm{E} S_{k, n}^{*}\right| \geqslant \varepsilon N_{k}^{p}\right\} \leqslant \frac{A_{m}}{\left(\varepsilon N_{k}^{p}\right)^{m}}\left(\left(\sum_{l=1}^{N_{k}} \mathrm{E} Z_{k, l}^{2}\right)^{m / 2}+\sum_{l=1}^{N_{k}} \mathrm{E}\left|Z_{k, l}\right|^{m}\right)
$$

где $Z_{k, l}=Y_{k, l}-\mathrm{E} Y_{k, l}$.

Заметим также, что по лемме 3.1 для любого $j \geqslant 1$ верно неравенство

$$
\frac{1}{N_{k}} \sum_{l=1}^{N_{k}} \mathrm{E}\left|Z_{k, l}\right|^{j} \leqslant 2^{j} \beta \mathrm{E}\left|Y_{k}\right|^{j}
$$

$\mathrm{K}$ тому же, $\mathrm{E} Y_{k}^{j}=N_{k}^{j p} \mathrm{P}\left\{|X|>N_{k}^{p}\right\}+\mathrm{E} X_{k}^{j}$. Значит,

$$
\begin{aligned}
& \mathrm{P}\left\{\max _{1 \leqslant n \leqslant N_{k}}\left|S_{k, n}^{*}-\mathrm{E} S_{k, n}^{*}\right| \geqslant \varepsilon N_{k}^{p}\right\} \\
& \quad \leqslant C\left(\left(N_{k} \mathrm{P}\left\{|X|>N_{k}^{p}\right\}\right)^{m / 2}+\left(\frac{\mathrm{E} X_{k}^{2}}{N_{k}^{2 p-1}}\right)^{m / 2}+N_{k} \mathrm{P}\left\{|X|>N_{k}^{p}\right\}+\frac{\mathrm{E} X_{k}^{m}}{N_{k}^{m p-1}}\right) .
\end{aligned}
$$

Обратимся к случаю а):

$$
\begin{aligned}
\int_{0}^{1} & u^{\alpha} \sum_{k=1}^{\infty} N_{k} \mathrm{P}\left\{|X| \geqslant u N_{k}^{p}\right\} d u=\int_{0}^{1} u^{\alpha} \mathrm{E} g\left(|X / u|^{1 / p}\right) d u \\
& \leqslant \int_{0}^{1} u^{\alpha} \mathrm{E} G\left(|X / u|^{1 / p}\right) d u+\int_{0}^{1} u^{\alpha} \mathrm{E}|X / u|^{1 / p} d u \\
& =C \int_{0}^{1} \frac{u^{\alpha}}{u^{2 / p}} \int_{0}^{\infty} F(x) \gamma\left(\left(\frac{x}{u}\right)^{1 / p}\right) x^{2 / p-1} d x d u+C \int_{0}^{1} u^{\alpha-1 / p} d u \\
& \leqslant C \int_{0}^{1} u^{\alpha-(2+\theta) / p} d u \int_{0}^{\infty} F(x) \gamma\left(x^{1 / p}\right) x^{2 / p-1} d x+C \int_{0}^{1} u^{\alpha-1 / p} d u
\end{aligned}
$$

Здесь мы использовали лемму 3.4 и условие (2). Таким образом, получаем для любого $\alpha>(2+\theta) / p$ сходимость ряда

$$
\sum_{k=1}^{\infty} N_{k} \int_{0}^{1} u^{\alpha} \mathrm{P}\left\{|X| \geqslant u N_{k}^{p}\right\} d u
$$

Интегрирование по частям дает

$$
\frac{\mathrm{E} X_{k}^{m}}{N_{k}^{p m-1}}=N_{k}^{1-p m} \int_{0}^{N_{k}^{p}} t^{m} d \mathrm{P}\{|X| \leqslant t\} \leqslant N_{k} m \int_{0}^{1} u^{m-1} \mathrm{P}\left\{|X| \geqslant u N_{k}^{p}\right\} d u .
$$


Из последнего неравенства и (12) получаем , что для любого $m>1+(2+\theta) / p$

$$
\sum_{k=1}^{\infty} \frac{\mathrm{E} X_{k}^{m}}{N_{k}^{p m-1}}<\infty
$$

Используя простое неравенство

$$
\mathrm{E} X_{k}^{2}=\mathrm{E} X^{2} \cdot I_{\left\{|X| \leqslant N_{k}^{p}\right\}} \leqslant \frac{N_{k}^{2 p}}{g\left(N_{k}\right)} \mathrm{E} g\left(|X|^{1 / p}\right)
$$

и условия (3) и (9), а также то, что $g\left(N_{k}\right)=\sum_{l=1}^{k} N_{l}$, легко установить неравенства:

$$
\sum_{k=1}^{\infty}\left(\frac{\mathrm{E} X_{k}^{2}}{N_{k}^{2 p-1}}\right)^{m / 2} \leqslant C \sum_{k=1}^{\infty}\left(\frac{N_{k}}{g\left(N_{k}\right)}\right)^{m / 2} \leqslant C \sum_{k=1}^{\infty} k^{-\delta m / 2}<\infty
$$

при $m \geqslant 2 / \delta$. Из (11), (13) и (14) следует, что утверждение теоремы в случае а) доказаHO.

Обратимся теперь к случаю б). Заметим, что (5) влечет существование $\Delta>0$ и $k_{0} \leqslant 1$ таких, что $N_{k+1} \geqslant \Delta \sum_{l=1}^{k} N_{l}, k \geqslant k_{0}$, откуда по индукции можно получить, что $N_{k} \geqslant c \lambda^{k}, k>k_{0}$, где $\lambda=1+\Delta>1$. Тогда для любого $j \geqslant 2$ имеем

$$
\begin{aligned}
\sum_{k=1}^{\infty} \frac{\mathrm{E}|X|^{j} \cdot I_{\left\{|X| \leqslant N_{k}^{p}\right\}}}{N_{k}^{j p-1}} & =\mathrm{E}|X|^{j} \cdot\left(\sum_{k=1}^{\infty} \frac{I_{\left\{|X| \leqslant N_{k}^{p}\right\}}}{N_{k}^{j p-1}}\right) \\
& \leqslant \mathrm{E}|X|^{j} \cdot\left(\sum_{k: N_{k} \geqslant X^{1 / p}} \frac{1}{N_{k}^{j p-1}}\right) \leqslant C \mathrm{E}|X|^{1 / p} .
\end{aligned}
$$

Применяя (18) с $j=2$, получим оценку $\sum_{k=1}^{\infty} \mathrm{E} X_{k}^{2} / N_{k}^{2 p-1}<\infty$, а значит, и для любого $m>2$

$$
\sum_{k=1}^{\infty}\left(\frac{\mathrm{E} X_{k}^{2}}{N_{k}^{2 p-1}}\right)^{m / 2}<\infty
$$

Таким образом, из (11), (16) и (15) при $j=m$ следует утверждение теоремы 2.1 в случае б).

ДОКАЗАТЕЛЬСТВО ТЕОРЕМЫ 2.2. Заметим, что

$$
\max _{1 \leqslant n \leqslant N_{k}}\left|X_{k, n}\right| \leqslant 2 \max _{1 \leqslant n \leqslant N_{k}}\left|S_{k, n}\right|
$$

Поэтому из (7) для любого $\varepsilon>0$

$$
\sum_{k=1}^{\infty} \mathrm{P}\left\{\max _{1 \leqslant n \leqslant N_{k}}\left|X_{k, n}\right| \geqslant \varepsilon N_{k}^{p}\right\}<\infty
$$


Свойство отрицательной определенности случайньх величин дает неравенство

$$
\mathrm{P}\left\{\max _{1 \leqslant n \leqslant N_{k}} X_{k, n} \leqslant t\right\} \leqslant \prod_{n=1}^{N_{k}} \mathrm{P}\left\{X_{k, n} \leqslant t\right\} \quad \text { для любого } t>0,
$$

так как функция $f(x)=1_{\{x \leqslant t\}}$ является невозрастающей, или, что то же самое,

$$
\mathrm{P}\left\{\max _{1 \leqslant n \leqslant N_{k}} X_{k, n} \geqslant t\right\} \geqslant 1-\prod_{n=1}^{N_{k}}\left(1-\mathrm{P}\left\{X_{k, n} \geqslant t\right\}\right) .
$$

Построим на некотором вероятностном пространстве набор независимых случайных величин $\left\{Z_{k, l}, 1 \leqslant l \leqslant N_{k}, k \geqslant 1\right\}$ таких, что величина $Z_{k, l}$ совпадает по распределению c $X_{k, l}$. Тогда $\mathrm{P}\left\{\max _{1 \leqslant n \leqslant N_{k}} Z_{k, n} \geqslant t\right\}=1-\prod_{n=1}^{N_{k}}\left(1-\mathrm{P}\left\{X_{k, n} \geqslant t\right\}\right)$ и, следовательно, $\sum_{k=1}^{\infty} \mathrm{P}\left\{\max _{1 \leqslant n \leqslant N_{k}} Z_{k, n} \geqslant \varepsilon N_{k}^{p}\right\}<\infty$. По лемме Бореля-Кантелли имеем

$$
\mathrm{P}\left\{\max _{1 \leqslant n \leqslant N_{k}} Z_{k, n} \geqslant \varepsilon N_{k}^{p} \text { бесконечно часто по } k\right\}=0 .
$$

Учитьвая, что величины $Z_{k, l}$ независимы в совокупности, еще раз применяем лемму Бореля-Кантелли:

$$
\sum_{k=1}^{\infty} \sum_{l=1}^{N_{k}} \mathrm{P}\left\{Z_{k, l} \geqslant \varepsilon N_{k}^{p}\right\}<\infty .
$$

Проведя аналогичные рассуждения для величин $\left\{-X_{k, n}, 1 \leqslant n \leqslant N_{k}\right\}$, которые также являются отрищательно ассоциированными величинами, получим утверждение теоремы 2.2.

ДокАЗАТЕЛЬСТвО ТЕОРЕМЫ 2.3. Достаточность легко следует из теоремы 2.1 и очевидного неравенства

$$
\max _{1 \leqslant n \leqslant N_{k}}\left|S_{n}^{(k)}\right| \leqslant\left|S_{k, N_{k}}\right|+\max _{1 \leqslant n \leqslant N_{k}}\left|X_{k, n}\right|,
$$

$\operatorname{aP}\left\{\max _{1 \leqslant n \leqslant N_{k}} X_{k, n} \geqslant \varepsilon N_{k}^{p}\right\} \leqslant N_{k} \mathrm{P}\left\{X_{k, n} \geqslant N_{k}^{p}\right\}$.

Докажем необходимость. Поскольку

$$
S_{k, N_{k}}=\frac{1}{N_{k}-1} \sum_{n=1}^{N_{k}} S_{n}^{(k)}
$$

то

$$
\left|S_{k, N_{k}}\right| \leqslant \frac{N_{k}}{N_{k}-1} \max _{1 \leqslant n \leqslant N_{k}}\left|S_{n}^{(k)}\right|
$$

Итак,

$$
\max _{1 \leqslant n \leqslant N_{k}}\left|X_{k, n}\right| \leqslant\left|S_{k, N_{k}}\right|+\max _{1 \leqslant n \leqslant N_{k}}\left|S_{n}^{(k)}\right| \leqslant\left(2+\frac{1}{N_{k}-1}\right) \max _{1 \leqslant n \leqslant N_{k}}\left|S_{n}^{(k)}\right| .
$$

Следовательно, из (8) следует (17). Дальнейшие рассуждения проводятся так же, как при доказательстве теоремы 2.2 .

Автор выражает благодарность профессору А.В. Булинскому за множество полезных замечаний, способствовавших улучшению данной статьи. 


\section{СПИСОК ЦИТИРОВАННОЙ ЛИТЕРАТУРЫ}

[1] Hsu P. L., Robbins H. Complete convergence and the law of large numbers // Proc. Nat. Acad. Sci. 1947. V. 33. P. 25-31.

[2] Erdös P. On a theorem of Hsu and Robbins // Ann. Math. Statist. 1949. V. 20. P. 286-291.

[3] Hu T.C., Moricz F., Taylor R. L. Strong laws of large numbers for arrays of rowwise independent random variables // Acta Math. Hungar. 1992. V. 54. P. 153-162.

[4] Asmussen S., Kurtz T. G. Necessary and sufficient conditions for complete convergence in the law of large numbers // Ann. Probab. 1980. V. 8. №1. P. 176-182.

[5] Gut A. On complete convergence in the law of large numbers for subsequences // Ann. Probab. 1985. V. 13. № 4. P. 1286-1291.

[6] Gut A. Complete convergence for arrays // Periodica Math. Hungar. 1992. V. 25. № 1. P. 51-75.

[7] Kuczmaszewska A., Szynal D. On the Hsu-Robbins law of large numbers for subsequences // Bull. Polish Acad. Sci. Math. 1988. V. 36. №1. P. 69-79.

[8] Bulinski A. V. Limit laws for arrays // Int. Congress of Mathematicians. Abstracts of communications. Berlin, 1998. P. 24.

[9] Bulinski A. V., Mikusheva A. E. SLLN and other laws for arrays // 7th Vilnius Conference on Probab. Theory. Abstracts of communications, 1998. P. 167.

[10] Микушева А. Е. Закон больших чисел и логарифомический закон в схеме серий // Фундамент. и прикл. матем. (в печати).

[11] Joag-Dev K., Proschan F. Negative association of random variables with applications // Ann. Statist. 1983. V. 11. P. 286-295.

[12] Su C. A theorem of Hsu-Robbins type for negatively associated sequence // Chinese Sci. Bull. 1996. V. 41. №6. P. 441-446.

[13] Su C., Zhao L., Wang Y. Moment inequality and weak convergence for negatively associated sequences // Sci. in China. Ser. A. 1997. V. 40. № 2. P. 172-182. 